Itinéraires Itinéraires

Littérature, textes, cultures

\title{
La théorie des imaginaires de la traduction
}

Introduction

\section{Christina Bezari, Riccardo Raimondo et Thomas Vuong}

\section{(2) OpenEdition}

Journals

Édition électronique

URL : http://journals.openedition.org/itineraires/5062

DOI : $10.4000 /$ itineraires.5062

ISSN : 2427-920X

Éditeur

Pléiade

Référence électronique

Christina Bezari, Riccardo Raimondo et Thomas Vuong, « La théorie des imaginaires de la traduction », Itinéraires [En ligne], 2018-2 et 3 | 2019, mis en ligne le 20 février 2019, consulté le 17 juin 2019. URL : http://journals.openedition.org/itineraires/5062 ; DOI : 10.4000/itineraires.5062

Ce document a été généré automatiquement le 17 juin 2019.

\section{cc) (i) $\ominus$}

Itinéraires est mis à disposition selon les termes de la licence Creative Commons Attribution - Pas d'Utilisation Commerciale - Pas de Modification 4.0 International. 


\title{
La théorie des imaginaires de la traduction
}

\author{
Introduction
}

Christina Bezari, Riccardo Raimondo et Thomas Vuong

[...] wie soll Erklärung auch nur möglich sein, wenn wir alles erst zum Bilde machen, zu unserem Bilde!

Friedrich Nietzsche, Die fröhliche Wissenschaft,

Aphorismus $\mathrm{n}^{\circ} 112$.

«[...] comment existerait-il même la possibilité d'expliquer quand nous faisons d'abord de toute chose une image, notre image!" Friedrich Nietzsche, Le gai savoir, trad. fr. par Alexandre Vialatte, $n^{\circ} 112$.

\section{Préambules}

1 À l'heure où le spectre de la traduction automatique promet à l'humanité des logiciels de plus en plus performants, il est important de rappeler que la traduction littéraire, la plus exigeante à de nombreux égards, reste un élément qui échappe aux algorithmes de l'intelligence artificielle. Non plus que l'écriture littéraire, la traduction n'est pas uniquement activité logique, où chaque mot d'une langue trouverait de manière automatique un équivalent dans une autre; de nombreux autres paramètres interviennent, certains à rebours de la prétention, ou simplement la tension, à la rationalité. Comment décrire donc la complexe constellation de facteurs impliqués dans les procédés traductifs? Comment rendre compte des profondeurs qui se dévoilent dans les processus interlinguistiques? La notion d'imaginaire aide à modéliser de nombreux éléments qui interviennent dans tout transfert culturel et notamment traductif.

2 Yves Bonnefoy conçoit l'imaginaire comme entité diffuse et disparate composée de «ces imaginations qui font la littérature » $(2013: 103)^{1}$. C'est là une première voix pour dire que l'être humain n'écrit et ne traduit pas qu'en fonction d'une approche linguistique qui 
serait rationnelle et systématique; sans doute un pan entier de la traduction a lieu en dehors de cette dimension logicielle. Comme le rappelle Novalis, la traduction littéraire est essentiellement une activité poétique qui requiert « la pratique de cet art d'imagination, de ce génie inventif» (Novalis [1798] 1975 : 374). La notion d'imaginaire évoque ainsi les dimensions de l'irrationnel et de l'imagination, autant que des référents culturels explicites comme implicites. Nous empruntons ce terme non seulement à la philosophie, à la psychologie et à l'anthropologie (Védrine 1990, Thomas 1998, Mary 2010, Mannoni 2016), mais aussi à la critique littéraire (Mitterand 2000), pour l'appliquer aux études traductologiques.

3 La notion d'imaginaire, qui nous a occupée's pendant deux journées d'études en $2016^{2}$, représente également un concept utile au développement des rapports entre traductologie et littérature comparée en ce qu'elle nous permet d'explorer la tension vers l'altérité qui les fonde. Elle peut agir comme révélateur heuristique de la complexité et des risques propres à ces disciplines hybrides, rendus nets par les travaux de Gayatri Spivak (2003) et Barbara Cassin (2016a). En effet, comme l'a avancé Gillian Lane-Mercier (2009), la littérature comparée et la traductologie trouveraient leur spécificité commune à la fois dans la visée centrifuge, nomade ou encore "cartographique " qui les anime, dans la logique de l'intersection, du réalignement, de la traversée. C'est par le biais d'un tel processus que ces deux disciplines deviennent des champs de tension mais aussi de synthèse.

4 Le recours à la notion d'imaginaire rend enfin nécessaire de considérer la traduction, non seulement du point de vue de la littérature, mais aussi de l'histoire des savoirs et des pratiques sociales (Rastier 2011, Guillaume 2014) qui la conditionnent. C'est ce à quoi nous nous proposons de participer ici, en développant des approches de la traduction au prisme de la philosophie, de la poétique, de la psychologie, c'est-à-dire de la repenser comme un art et non comme l'un des domaines de la linguistique appliquée.

5 En consacrant un numéro de la revue Itinéraires à la théorie des imaginaires de la traduction, nous souhaitons placer les études traductologiques en perspective avec d'autres domaines des sciences humaines et sociales, en nourrissant une démarche comparatiste et cartographique. Pour ce faire, il faut préalablement se pencher sur la notion d'imaginaire en traduction, tant elle recouvre un domaine délicat à définir, mais d'autant plus nécessaire à une époque où l'hybridation des savoirs devient de plus en plus une urgence scientifique.

\section{Esquisses définitoires sur l'imaginaire en traduction}

6 De nombreux paramètres permettent de décrire l'ensemble des caractéristiques d'une traduction, y compris en sondant au-delà et en deçà du texte traduit; il est tentant de chercher un nœud liant la constellation de termes couramment utilisés en traductologie comme visée du traducteur, projet de traduction, récit de traduction, orientation du texte traduit, geste traductif, etc. Qu'il s'agisse d'un projet de traduction explicite ou implicite, du paratexte du texte source ou du texte cible, des affinités littéraires apparentant le traducteur et son modèle, etc., on peut facilement s'apercevoir que la dimension textuelle dialogue avec une dimension autre qu'on appellera ici imaginaire.

Gilbert Durand, dans une perspective anthropologique, définissait l'imaginaire comme le lieu des échanges «entre les pulsions subjectives et assimilatrices et les intimations 
objectives émanant du milieu cosmique et social» (Durand 1969 : 38). L'imaginaire serait donc « ce trajet dans lequel la représentation de l'objet se laisse assimiler et modeler par les impératifs pulsionnels du sujet, et dans lequel réciproquement [...] les représentations subjectives s'expliquent par les accommodations antérieures du sujet au lien objectif » ( ibid.). D'un point de vue traductologique, on peut ainsi définir l'imaginaire comme la dimension dans laquelle les conceptions de la traduction, les interprétations du textesource et les processus interlinguistiques (objets) sont assimilés, modelés et modélisés par les traducteurs (sujet).

On peut aussi s'inspirer, dans ce contexte, des travaux récents de Graham Harman (2018) qui montrent que des représentations idéologiques et fictionnelles constituent des faits, des conditions concrètes de tout acte humain. L'imaginaire peut donc être envisagé comme l'ensemble des représentations qui façonnent la perception humaine du monde. Ces représentations peuvent être conscientes ou non ; subies ou choisies; prises à travers une optique singulière ou des projections collectives; clichés, archétypes, influences intertextuelles, métaphores, images fixes ou mouvantes, etc. Quelle que soit leur nature, elles constituent le prisme grâce auquel l'esprit humain appréhende un objet, au point que ces représentations mêmes s'animent d'une vitalité propre.

En traductologie, une étude de l'imaginaire consiste donc à sonder les modalités par lesquelles ce prisme module et dynamise la traduction, envisagée à la fois comme notion en soi (ex. les conceptions de la traduction) et comme processus (l'acte du traduire et les pratiques traductives). On pourrait ainsi résumer les approches des imaginaires de la traduction à travers deux principaux volets: les imaginaires du traduire et les imaginaires des traducteurs et traductrices.

\section{Imaginaires du traduire}

Dans un premier temps, il est intéressant d'observer les manières par lesquelles, comme l'a avancé le premier Antonio Lavieri, l'imaginaire intervient dans la "réélaboration socio-symbolique des pratiques traductionnelles» (Lavieri 2010: 120). On parlera donc d'un "imaginaire du traduire», qui peut s'incarner ainsi dans des métaphores, des stéréotypes, des «mythes du traduire» ou des récits de traduction (Delabastita et Grutman 2005 ; Hagedorn 2006 ; Lavieri 2007, 2010). Si Hagedorn analysait principalement les caractéristiques des pseudotraductions narratives (de Cervantès à Calvino en passant par Montesquieu, Walpole, Potocki, Wieland ou Schmidt), Antonio Lavieri, quant à lui, a mis l'accent sur le "pouvoir heuristique» de la fiction et sur la possibilité (offerte par l'épistémologie des sciences) d'étendre «le modèle fictionnel de la science moderne à tout système symbolique » (Lavieri $2007: 16$ ).

11 Un imaginaire du traduire sera composé ainsi de l'ensemble des représentations du processus traductif et du système symbolique qui en dérive (à une époque spécifique et dans une culture donnée), tout en incluant les connotations appliquées à l'acte de traduction dans les textes théoriques ou dans les paratextes.

\section{Imaginaire des traducteurs et traductrices}

Dans un second temps, il est crucial d'observer les procédés par lesquels l'imaginaire subjectif de qui traduit, en relation avec l'imaginaire collectif, joue un rôle concret dans la 
pratique traductive, dans des choix linguistiques et poétiques (Raimondo 2016a, 2016b). La traductologie peut ainsi consister en une " génétique de la traduction » au sens large qui repense son identité à la lumière des études sur l'imaginaire. En d'autres termes, il s'agit de développer la compréhension de ce que Berman a appelé «horizon traductif » envisagé comme «l'ensemble des paramètres langagiers, littéraires, culturels et historiques qui "déterminent" le sentir, l'agir et le penser d'un traducteur » (Berman 1995 : 79-83).

On peut ainsi considérer la notion d'imaginaire des traducteurs comme une déclinaison des théories sur «l'imaginaire linguistique " (ex. Houdebine 1979, 2002, 2015 ; Moreau 1998 ; Glissant 2010 ; Pot 2018), notion élaborée par Anne-Marie Houdebine dans le cadre de sa thèse d'État (1975-1979). On peut également concevoir la notion d'imaginaire de la traduction comme l'un des possibles paradigmes de "l'imaginaire culturel », notion brièvement traitée, mais jamais modélisée, par Houdebine (1940-2016) comme «l'une des extensions de l'imaginaire linguistique » et qui peut aussi s'entendre comme imaginaire «historique et social» (Houdebine 2015). La traduction serait par ailleurs, selon Houdebine, « un lieu privilégié pour traiter de l'imaginaire linguistique et l'imaginaire culturel, puisque deux langues et partant deux cultures a minima sont alors en cause : celle de l'auteur, celle du traducteur, de leurs époques, de leurs lectures, etc.» (Houdebine 2015 : 29). Si l'imaginaire linguistique peut être défini comme rapport du sujet à la langue (Moreau 1998 : 165-167) ou plus précisément comme « le rapport du sujet à sa langue intime et à la langue commune (la langue)" (Houdebine 2015: 18), l'imaginaire des traducteurs concernera le rapport du sujet à tous les processus impliqués dans la traduction ainsi qu'aux conceptions et aux imaginaires du traduire.

Il s'agit enfin d'émettre des hypothèses sur la psyché des traducteurs, sur la manière dont elle agit face aux textes sources. Avec une certaine prudence, on pourra ainsi lier la théorie des imaginaires à la "psychologie de la traduction » (Jääskeläinen 2012). Les travaux de nos devanciers, comme ceux de Linda Collinge (2000) ou Mathias Verger (2010), n'offrent pas une définition exacte de la notion d'imaginaire en traduction et, plus particulièrement, de celle plus complexe d'imaginaire des traducteurs ; c'est pourquoi il convient de préciser cette notion.

\section{Imaginaire et imagination}

15 La difficulté de la notion d'imaginaire fait aussi sa richesse: son caractère transdisciplinaire et hybride, à la lisière entre psychologie, littérature et linguistique, la rend protéiforme et fugace.

On peut encadrer les processus traductifs à l'intérieur de ce qu'on pourrait appeler les « circonstances événementielles de la production imaginaire " (Van Eynde 2005 : 9). Cela revient à dire que pratiques et gestes traductifs ne peuvent être réduits à de simples processus logico-grammaticaux, mais nécessitent une réflexion plus profonde portant sur la subjectivité des traducteurs, sur leur production imaginaire.

On observera facilement que nombre de choix traductifs dérivent de la créativité des traducteurs. Empruntant une notion jungienne, on parlera alors d'une «imagination active » (Jung [1954] 1998 : 1046) qui s'incarne, consciemment ou inconsciemment, dans des choix linguistiques et poétiques: on peut parler aussi de la "disposition germinative » (Jung [1956] 1998: 330) de l'imagination traductive, c'est-à-dire de son 
caractère créatif. Cette conception de l'imagination fait clairement écho à celle de Giambattista Vico qui, dans la Scienza nuova $(1725,1730$ et 1744 ; ici éd. 2012) exposait la doctrine des « universaux fantastiques» (universali fantastici) dans laquelle l'imagination est considérée par rapport à son lien avec la poésie (en tant que poiesis, en gr. roínoıs, " création ») et avec la transformation des formes du savoir exprimée par la société dans l'histoire.

L'imaginaire - comme lieu de représentation de la réalité et, par conséquent, de production de savoirs - est profondément lié à l'imagination. L'imaginaire des traducteurs peut alors être défini comme la constellation d'éléments réels et abstraits qui constituent la psyché du traducteur et influencent son imagination traductive.

Bien évidemment, nous n'avons pas l'ambition de réinventer une «théorie de l'imagination » à la manière de Paul Ricœur (1986: 237 et passim), mais proposons plutôt un examen des différentes composantes de l'esprit qu'il nomme " poétique de la volonté " (ibid.) permettant d'observer un certain nombre de phénomènes et d'expériences «à la charnière du théorique et du pratique » (Ricœur 1986:238). Une telle investigation peut offrir aux lecteurs quelques réflexions sur cette «entité mentale", cette "étoffe dans laquelle nous taillons nos idées abstraites, nos concepts », cet "ingrédient de je ne sais quelle alchimie mentale » (Ricœur $1986: 241$ ), c'est-à-dire l'imagination et l'ensemble des éléments de son arrière-plan constituant l'imaginaire. Cette approche ouvre un nouveau champ d'études permettant d'émettre des hypothèses sur les émotions qu'éprouvent les traducteurs, les conceptions et les imaginaires du traduire qu'ils partagent, leur activité imaginative lorsqu'ils interprètent le texte-source.

Cette conception particulière de l'imaginaire, bien qu'inhabituelle et pionnière en traductologie, n'est pas la première dans le panorama des sciences humaines. On peut par exemple renvoyer aux études d'olivier Rimbault (2016:24-28 et passim) qui, reprenant les théories de Carl Gustav Jung ([1950] 1993 : 24-27) et de Gilbert Durand (1969), envisage l'existence d'une structure au fondement à la fois de l'imaginaire et des discours culturels qui en dérivent: une "zone matricielle" (Jung [1950] 1993: 435) à l'origine des archétypes et des idées.

21 Peut-on, de ce point de vue, parler d'imaginaires matriciels de la traduction? Dans quelle mesure les imaginaires culturels (socio-politiques, religieux, spirituels, identitaires, etc.) influencent-ils les imaginaires du traduire et les imaginaires des traducteurs? Les contributions présentées dans ce volume par ordre thématique offriront des réponses à ces questionnements comme à d'autres qui ont été posés par le caractère spécifique de chaque article.

\section{Parcours}

Les deux grands champs prospectifs de l'étude de l'imaginaire des traductions - imaginaire du traduire et imaginaire des traducteurs - ne s'étendent pas séparément, en autonomie; ils se recoupent et se nourrissent. Afin de cheminer dans les sylves foisonnantes de l'imaginaire, nous avons ordonné les contributions depuis les plus théoriques aux plus pragmatiques. Tout en distinguant les deux volets de notre théorie à l'intérieur des parcours, ce numéro s'ouvre par des pistes de réflexion sur les conceptions de la traduction, avant de poursuivre par des études de cas plus précises. Le volume se 
termine par deux témoignages de traductrices, deux récits de traduction qui convoquent la notion d'imaginaire dans la pratique traductive et dans son contexte.

fictionnelle, Marta Álvarez Izquierdo emploie ce concept afin de l'appliquer, sous le signe de la perte, à l'étude de la poétique de l'ambiguïté chez le romancier uruguayen Juan Carlos Onetti. L'imaginaire de l'auteur se trouve perdu et profondément affecté par le processus traductif, témoignant de la nécessité d'intégrer cette dimension de l'écriture à la traduction.

Les quatre premières contributions abordent la notion d'imaginaire en traduction du point de vue des théories et des représentations du traduire, montrant à la fois leur versatilité et leur ancrage profond, qui les rendent impossibles à exclure d'une étude traductologique.

En ouverture, Christine Lombez propose une réflexion sur les influences du geste traductif, en y étudiant le rôle de l'imaginaire, confirmant notre prémisse d'irréductibilité de la traduction à une simple mécanique. Soulignant qu'on traduit toujours avec-les œuvres du passé, leur interprétation, les interférences du contemporain, etc.-Christine Lombez démontre la prégnance de ces partenariats, conscients ou non. Si son approche concerne en particulier le cas de la poésie, elle démontre l'absolue nécessité d'étudier ces dimensions de l'imaginaire qui informent la traduction.

Se concentrant sur les techniques du travail traductif, Paolo Bellomo s'intéresse à un procédé emblématique des ambiguïtés théoriques de la traduction : le calque, dont il trace une histoire théorique. Si l'on dépasse ce qu'on croit connaître du procédé, pour suivre par exemple Walter Benjamin et Georges Didi-Huberman, le calque s'avère contenir en sous-texte des valeurs parfois opposées à l'acception contemporaine de fidélité fautive.

Au-delà du calque même, Solange Arber démontre l'importance des imaginaires idéologiques et politiques dans la conception du traduire en y étudiant la prétention - fantasmatique ? - à la transparence, tension entre imaginaire et procédés linguistiques. On relèvera en particulier le geste traductif réalisé en vitrine, suivant l'intuition provocante d'Emil Tophoven, qui éclaire toute une réflexion sur l'imaginaire de la traduction comme transparence.

C'est ce que confirme Aude A. Gwendoline, qui s'intéresse à la dualité entre blessure et guérison en opposant les métaphores du traduire comme violence dans les pratiques traductives d'Artaud, de Nancy Huston, de Louis Wolfson ou d'Anna O., qui la reçoivent comme un bienfait. Le tiraillement entre deux imaginaires du traduire recoupe ici le contraste entre théorie et pratique, imaginaire du traduire et effet de la traduction sur l'imaginaire, les conceptions du traduire et les pratiques traductives.

Après ces investigations théoriques démontrant l'importance épistémologique de la notion d'imaginaire, les contributions suivantes montrent comment elle peut être appliquée à l'étude d'un corpus précis. Il peut s'agir aussi bien d'imaginaires du traduire que des imaginaires des traducteurs, tant les deux sont liés. Ces études montrent l'étendue de l'influence de l'imaginaire, appliqué à l'analyse textuelle : pertes de sens et germinations sémantiques, superpositions et substitutions, opérations linguistiques conscientes et actes créatifs inconscients, etc.

Passant ainsi à l'étude de traducteurs et au rôle de leurs traductions dans leur pratique Montrant en quelle mesure ces notions peuvent s'appliquer à l'étude de romanciers canoniques, Lilia Androsenko consacre sa contribution au rôle de la traduction de Balzac 
par le jeune Dostoïevski. Cette version française nous permet à la fois d'observer les différents mécanismes de transposition d'éléments du texte source en fonction des imaginaires (de l'auteur comme du traducteur), et d'envisager une influence de cette traduction de jeunesse dans le parcours à venir du romancier russe.

Dans une perspective inverse, Laude Ngadi Maïssa se penche sur le cas de la traduction des œuvres d'Olivier Rolin, pour en établir une carte analytique. Cette cartographie, appuyée sur des statistiques éditoriales et des entretiens avec les traducteurs de Rolin, témoigne de la capacité d'une œuvre complexe et foisonnante à faire surgir divers imaginaires nationaux et linguistiques, la notion servant ainsi de pierre de touche pour l'étude de la traduction comme réception.

Mêlant à cette approche de la réception une étude sur les conceptions de la traduction chez des auteurs de fiction, Louis Watier, dans le sillage de Hagedorn, s'intéresse aux imaginaires du traduire dans des textes qui se font passer pour traductions, et montre que ceux-ci, au XVI ${ }^{\mathrm{e}}$ siècle, contribuent à la définition de la notion même de fiction. Dans ce cas, l'imaginaire du traduire dépasse le cadre du seul traducteur pour devenir imaginaire collectif et pour jouer un rôle considérable dans l'histoire littéraire.

Gloria Branca choisit d'examiner la spécificité d'une écriture migrante, entre deux pays et deux langues, en se concentrant sur le cas de Cioran et de sa traduction en italien: traduire le français de Cioran est non seulement un défi que se lance son traducteur italien, Mario Andrea Rigoni, mais également un révélateur des imaginaires linguistiques tant de l'auteur que de son traducteur.

34 La contribution d'El-Shaddai Deva offre quant à lui un autre type de perspective plurilingue, en étudiant les traductions allemandes des œuvres du romancier camerounais Ferdinand Oyono, dans lesquelles on peut apprécier différentes variétés du français, comme l'idiome appelé "petit-nègre » influencé par les langues indigènes des anciennes colonies françaises. L'imaginaire linguistique pluriel de cet auteur pose différents défis, auxquels diverses stratégies traductives tentent de répondre en révélant notamment le gouffre entre les imaginaires coloniaux propres aux deux espaces linguistiques.

On peut facilement comparer cette analyse avec celle, plus ciblée, que Marianne Braux consacre aux différentes traductions d'un roman de Lydie Salvaire, Pas pleurer, qui traitent différemment la langue espagnole et les imaginaires sociopolitique et linguistique qui lui sont associés. Ce faisant, Braux nous offre une démonstration de l'importance de la notion d'imaginaire dans les études sur l'hétérolinguisme.

Les riches perspectives des parcours ainsi définis demeurent dans le domaine de la recherche littéraire et historico-culturelle. En revanche, les deux dernières contributions sont le résultat à la fois d'un questionnement théorique et de l'expérience pratique de deux chercheuses-traductrices. Elles explorent la dimension de l'imaginaire dans le processus traductif qui les a impliquées.

37 Frances Egan interroge directement les écartèlements des imaginaires en évoquant son expérience de la traduction d'un roman d'Alexandre Vialatte, au sujet duquel elle se demande comment elle peut se positionner en tant qu'Australienne du $\mathrm{xxI}^{\mathrm{e}}$ siècle. À travers ce passionnant récit de traduction, la traductrice et la chercheuse nous donnent ainsi l'opportunité de scruter dans leurs imaginaires. Comme dans un jeu de miroir, la chercheuse analyse la traductrice, se questionne sur sa conception de la traduction et élabore des stratégies pour sa pratique traductive. 
Enfin, l'approche d'Irène Gayraud permet de compléter cette cartographie des influences de l'imaginaire sur la traduction, en évoquant une démarche novatrice, celle qui consiste, dans le sillage de l'Oulipo, à lier traduction et potentialité. Ce faisant, ce sont les différentes virtualités du texte, et les divers imaginaires qui peuvent y abonder, qui sont explorés par la traduction, qui ouvrent le potentiel de l'œuvre. Gayraud nous invite à visiter son atelier traductif en reparcourant son expérience personnelle liée à une conception de la traduction courageuse et iconoclaste.

Les interventions ici réunies nous montrent donc que l'imaginaire constitue une notion épistémologique capitale: non seulement à la source de la démarche initiale de la traduction, mais aussi de sa réception comme de son étude littéraire et historique. Prenant pour point de départ des traditions linguistiques et culturelles différentes (ici France, Russie, Allemagne, Roumanie, Espagne, Canada, Uruguay, Afrique postcoloniale, etc.), et en contextualisant les imaginaires du traduire propres à chaque traducteur ou traductrice, ces contributions permettent de dessiner les contours mouvants de l'imaginaire opérant dans toute traduction, et ce quelles qu'en soient les facettes: culturelles, sociopolitiques, spirituelles, linguistiques, psychologiques, esthétiques, idéologiques, etc. Il devient également possible de tracer des catégories, des types de traducteurs et traductrices, grâce à leurs imaginaires.

Au-delà de cette approche des esprits traduisants, la notion d'imaginaire éclaire les concepts classiques de la traductologie, en les reliant à des imaginaires du traduire : sans doute faut-il évoquer les notions de transparence, de traduction sourcière ou cibliste, etc., en reliant les conceptions du traduire afférentes aux résultats textuels. Ces contributions démontrent que l'un ne va pas sans l'autre.

\section{Quelques implications de la notion d'imaginaire dans les théories de la traduction}

Plus largement, la théorie des imaginaires de la traduction peut agir comme élément vivifiant pour le développement, voire le renouvellement, des théories contemporaines de la traduction.

Premièrement, la notion d'imaginaire ouvre des voies pour une réflexion plus profonde sur la communication interculturelle, qui acquière une importance croissante dans le système d'échanges mondialisés (Olohan 2000). Le traducteur facilite les coopérations transnationales et agit en tant que médiateur interculturel visant à privilégier le dialogue entre communautés qui ne partagent pas forcément les mêmes imaginaires. De ce point de vue, si la réception du texte traduit est essentiellement un acte de communication implicite entre - au moins - deux langues et cultures qui interagissent et s'enrichissent mutuellement (House et Blum-Kulka 1986 : 12), c'est à travers ce processus d'interaction interlinguistique et interculturelle que les imaginaires du traduire et des traducteurs se multiplient et se concrétisent.

3 Toutefois, la traduction n'est pas simplement vue comme une façon de communiquer avec autrui ; elle est aussi un dispositif herméneutique (Ricœur 2016) qui nous aide à comprendre et à déchiffrer la réalité (Bezari $2018: 276)$. On sait depuis George Steiner que " comprendre, c'est déchiffrer » et qu' entendre une signification, c'est traduire » (Steiner 1975: 15). Selon cette formule, la traduction devient une langue intermédiaire qui unit la communication à l'entendement: le processus de traduction est considéré 
comme un langage en soi, un «troisième code » entre deux langues ou, si l'on veut, un "langage hybride» (hybrid language), selon une célèbre définition de William Frawley (1984). Comme dans la technique du carottage, la théorie des imaginaires de la traduction est un outil qui aide non seulement à explorer la surface horizontale de cette troisième langue, mais aussi à sonder ses profondeurs, son étendue verticale (projets de traduction, référents historico-culturels, conceptions du traduire, etc.).

Deuxièmement, si l'on envisage l'existence d'imaginaires matriciels de la traduction, on pourrait émettre l'hypothèse qu'ils constituent des universels de la traduction-notion inventée par Mona Baker dans une étude sur les corpus linguistiques (1993) ayant comme but d'illustrer des constantes dans le domaine du processus traductif. Cette notion, qui n'a pas connu beaucoup de succès dans le monde francophone, a été pourtant traitée par quelques auteurs français (ex. Gile 2015) et a été définie ainsi par Sara LaviosaBraithwaite :

Universals of translation are linguistic features which typically occur in translated rather than original texts and are thought to be independent of the influence of the specific language pairs involved in the process of translation.

Les universels de la traduction sont des caractéristiques linguistiques qu'on peut habituellement observer dans les textes traduits, plutôt que les originaux, et qui sont pensées comme indépendantes de l'influence des couples de langues spécifiques impliquées dans le processus traductif. (Laviosa-Braithwaite 1998: 288, nous traduisons)

Si pour Mona Baker les universels de la traduction concernent uniquement le domaine des processus linguistiques et textuels, la théorie des imaginaires de la traduction permet d'étudier aussi la dimension translinguistique et transtextuelle, et peut ainsi jouer le rôle de dispositif théorique et opérationnel pour étendre le champ des «universels » en traductologie.

Troisièmement, la théorie des imaginaires de la traduction se révèle particulièrement efficace pour conceptualiser des processus historiques et transhistoriques, des récurrences et des résurgences dans l'élaboration des conceptions du traduire, des évolutions et des modifications dans l'histoire des pratiques traductives. De ce point de vue, la notion d'imaginaire ouvre aussi des nouvelles voies pour le développement, non seulement de l'histoire des traductions (Pym 1998) et de l'histoire comparée des traductions (Coldiron 2001), mais aussi de ce qu'on peut désormais considérer comme le champ de recherche de la traductologie comparée (Tyulenev et Zheng 2017).

Quatrièmement, une enquête autour de l'imaginaire en traduction ne pourrait qu'étendre et enrichir le domaine de la psychologie. Si comme on l'a déjà évoqué, cette notion appartient déjà au champ des études psychologiques et anthropologiques, elle peut devenir un outil opératoire pour modéliser ces processus déjà étudiés et observés par la psychologie de la traduction (Jääskeläinen 2012): imagination et pensée imaginative, créativité, personnalité des traducteurs, apprentissage de la traduction, etc.

Plus généralement, s'intéresser à l'imaginaire des traductions et des traducteurs, c'est travailler à une meilleure compréhension de l'autre dans un monde que les traductologues (Cassin 2016a: 30; 2016b), les poètes (Bonnefoy 2013: 286-288) et les romanciers (Menasse 2017) présentent de manière prégnante comme celui d'après Babel. C'est une manière de donner aux lecteurs et aux lectrices des outils théoriques pour apprivoiser la complexité du texte traduit, pour s'interroger au quotidien sur le rôle de la traduction dans notre réalité, où la place croissante du multilinguisme ne saurait être laissée à des machines sans rêves. 


\section{BIBLIOGRAPHIE}

Baker, Mona, 1993, « Corpus Linguistics and Translation Studies - Implications and

Applications ", dans M. Baker, G. Francis et E. Tognini-Bonelli (dir.), Text and Technology. In Honour of John Sinclair, Amsterdam, John Benjamins, p. 233-250.

Berman, Antoine, 1995, Pour une critique des traductions : John Donne, Paris, Gallimard.

Bezari, Christina, 2018, «Figures du poète-traducteur : Friedrich Hölderlin et Gérard de Nerval », Australian Journal of French Studies, vol. 55, n 3, p. 274-287.

Bonnefoy, Yves, 2013, L'Autre Langue à portée de voix, Paris, Seuil.

Cassin, Barbara, 2016a, Éloge de la traduction, Paris, Fayard.

Cassin, Barbara (dir.), 2016b, Après Babel, traduire, Arles, Actes Sud.

Coldiron, Anne, 2011, « Toward A Comparative New Historicism: Land Tenures and Some Fifteenth-Century Poems », Comparative Literature, vol. 53, n² 2, p. 97-116.

Collinge, Linda, 2000, Beckett traduit Beckett : de « Malone meurt » à « Malone Dies », l'imaginaire en traduction, Genève, Droz.

Delabastita, Dirk et Grutman, Rainer (dir.), 2005, Fictionalising Translation and Multilingualism, Anvers, Hoger Instituut voor Vertalers \& Tolken.

Durand, Gilbert, 1969, Les Structures anthropologiques de l'imaginaire, Paris, Bordas.

Frawley, William, 1984, « Prolegomenon to a Theory of Translation », dans W. Frawley (dir.), Translation: Literary, Linguistic and Philosophical Perspectives, Londres, Associated University Press, p. 159-175.

Gile, Daniel, 2015, La Traduction. La comprendre, l'apprendre, Paris, PUF.

Glissant, Édouard, 2010, L'Imaginaire des langues, entretiens avec Lise Gauvin, Paris, Gallimard. Guillaume, Astrid, 2014, « Vers une sémiotique diachronique et contrastive des cultures », dans D. Ablali, S. Badir et D. Ducard (dir.), Documents, textes, œuvres. Perspectives sémiotiques, Rennes, PUR, p. 381-406.

Hagedorn, Hans Christian, 2006, La traducción narrada: el recurso narrativo de la traducción ficticia, Cuenca, Ediciones de la Universidad de Castilla-La Mancha.

Harman, Graham, 2018, Object-Oriented Ontology: A New Theory of Everything, Londres, Pelican.

Houdebine, Anne-Marie, 1979, La variété et la dynamique d'un français régional : étude phonologique, analyse des facteurs de variation, à partir d'une enquête à grande échelle dans le département de la Vienne (Poitou), thèse soutenue à l'Université Paris Descartes sous la direction d'André Martinet.

Houdebine, Anne-Marie (dir.), 2002, L'Imaginaire linguistique, Paris, L'Harmattan.

Houdebine, Anne-Marie, 2015, « De l'imaginaire linguistique à l'imaginaire culturel », $L a$ linguistique, $\mathrm{n}^{\circ}$ 51, p. 30-40.

House, Juliane et Blum-Kulka, Shoshana (dir.), 1986, Interlingual and Intercultural Communication. Discourse and Cognition in Translation and Second Language Acquisition Studies, Tübingen, Narr. 
Jääskeläinen, Riitta, 2012, « Translation Psychology », dans Y. Gambier et L. van Doorslaer (dir.), Handbook of Translation Studies, vol. 3, Amsterdam, John Benjamins, p. 191-197.

Jung, Carl Gustav, [1950] 1993, Types psychologiques, Préface et traduction par Y. Le Lay, 8 éd., Genève, Georg.

Jung, Gustav, [1956] 1998, Énergétique psychique, trad. par Y. Le Lay ; rééd. « L'énergétique psychique ", dans La Réalité de l'âme, 2 vol., éd. M. Cazenave, Paris, Librairie Générale Française, t. I.

Jung, Carl Gustav, [1954] 1998, « Réflexions théoriques sur la nature du psychisme », dans La Réalité de l'âme, 2 vol., éd. de Michel Cazenave, Paris, Librairie Générale Française, t. I.

Lane-Mercier, Gillian, 2009, « Repenser les rapports entre la littérature comparée et la traductologie : prolégomènes au braconnage interdisciplinaire », TTR : traduction, terminologie, rédaction, vol. 22, $\mathrm{n}^{\circ}$ 2, p. 151-182.

Lavieri, Antonio, 2007, Translatio in fabula. La letteratura come pratica teorica del tradurre, Rome, Editori Riuniti.

Lavieri, Antonio, 2010, « Homo translator. Notes pour une anthropologie comparative de la traduction ", dans S. Klimisi, I. Ost et S. Vanasten (dir.), Translatio in fabula. Enjeux d'une rencontre entre fictions et traductions, Bruxelles, Publications des facultés universitaires Saint-Louis, p. 117-127.

Laviosa-Braithwaite, Sara, 1998, « Universals of Translation », dans M. Baker et K. Malmkjær (dir.), Routledge Encyclopedia of Translation Studies, Londres, Routledge, p. 288-291.

Mannoni, Pierre, 2016, Les Représentations sociales, Paris, PUF.

Mary, André, 2010, « Imaginaire / Imaginaires », dans R. Azria et D. Hervieu-Leger (dir.), Dictionnaire des faits religieux, Paris, PUF, p. 524-529.

Menasse, Robert, 2017, Die Haupstadt, Berlin, Suhrkamp.

Mitterand, Henri, 2000, L'Imaginaire littéraire : des archétypes à la poétique du sujet, Paris, Nathan.

Moreau, Marie-Louise, 1998, Sociolinguistique (dictionnaire de), Concepts de base, Bruxelles, Mardaga. Nietzsche, Friedrich, [1882] 1950, Le Gai Savoir, trad. fr. par Alexandre Vialatte, Paris, Gallimard. Novalis [Hardenberg, Friedrich von], [1798] 1975, Grains de pollen, dans CEuvres complètes, édition établie, traduite et présentée par Armel Guerne, Paris, Gallimard.

Olohan, Maeve (dir.), 2000, Intercultural Faultlines: Research Models in Translation Studies, Manchester, St. Jerome Publishing.

Pot, Olivier (dir.), 2018, Langues imaginaires et imaginaire de la langue, Genève, Droz.

Pym, Anthony, 1998, Method in Translation History, Manchester, St. Jerome Publishing.

Raimondo, Riccardo, 2016a, « Le démon fugitif de l'imagination : propositions pour une traductologie comparée, entre Nerval et Baudelaire ", Nouvelle Fribourg, nº 2, [En ligne], http:// www.nouvellefribourg.com/archives/le-demon-fugitif-de-limagination-propositions-pour-unetraductologie-comparee-nerval-et-baudelaire/.

Raimondo, Riccardo, 2016b, « Orphée contre Hermès : herméneutique, imaginaire et traduction (esquisses)» Meta, $\mathrm{n}^{\circ}$ 61, p. 650-674, [En ligne], https://id.erudit.org/iderudit/1039223ar.

DOI : $10.7202 / 1039223 \mathrm{ar}$

Rastier, François, 2011, La Mesure et le Grain. Sémantique de corpus, Paris, Champion. 
Ricœur, Paul, 1986, Du texte à l'action, Essais d'herméneutique II, Paris, Seuil.

Ricœur, Paul, 2016, Sur la traduction, Paris, Les Belles Lettres.

Rimbault, Olivier, 2016, Imaginaire et pensée. Désiré Érasme, Martin Luther, Nicolas de Cues : trois imaginaires, trois modèles de pensée, Perpignan, Presses universitaires de Perpignan.

Spivak, Gayatri Chakravorty, 2003, Death of a Discipline, New York, Columbia University Press. Steiner, George, 1975, After Babel: Aspects of Language and Translation, Oxford, Oxford University Press.

Thomas, Joël, 1998, Introduction aux méthodologies de l'imaginaire, Paris, Ellipses.

Tyulenev, Sergey et Zheng, Binghan, 2017, « Toward Comparative Translation and Interpreting Studies ", Translation and Interpreting Studies, vol. 12, n 2, p. 197-212.

Van Eynde, Laurent, 2005, « Avant-propos », dans E. D’Arcier, J. Madou et L. Van Eynde (dir.), Mythe et création. Théorie et figures, 2 vol., t. I, Bruxelles, Publications des facultés universitaires Saint-Louis.

Védrine, Hélène, 1990, Les Grandes Conceptions de l'imaginaire : de Platon à Sartre et Lacan, Paris, Librairie générale française.

Verger, Mathias, 2015, « Antonin Artaud et l'imaginaire de la traduction », Carnets de Chaminadour , no 4, p. 61-85.

Vico, Giambattista, [1725, 1730 and 1744] 2012, Scienza nuova, éd. de Manuela Sanna et Vincenzo Vitiello, Milan, Bompiani, "Libro primo" (Dello stabilimento de' principi), § XLIX ; “Libro secondo" ( Della sapienza poetica), § V; "Libro quarto" (Del corso che fanno le nazioni), § VI.

\section{NOTES}

1. Il faut noter qu'Yves Bonnefoy exclut de la littérature et de ses rapports à l'imaginaire l'écriture poétique, selon la définition qu'il livre de ses buts ; si dans ce numéro Christine Lombez traite ce sujet précis, la plupart des études recueillies se concentrent bien sur les autres genres littéraires.

2. Les Journées d'études internationales « Les imaginaires de la traduction » les 3 et 4 mars 2017 à l'université Sorbonne-Nouvelle, pour lesquelles nous tenons à remercier le Campus Condorcet, le CERLIM, l'université de Gand, l'Équipe d'accueil Pléiade et la Commission de la Recherche de l'université Paris 13, et en particulier Mmes Bérénice Waty et Sandrine Francisco et les professeurs Pierre Civil et Jean-Yves Masson.

\section{RÉSUMÉS}

Une traduction ne peut se résumer à un échange clair et univoque de signes, qui seraient automatiquement remplaçables l'un par l'autre. La notion d'imaginaire permet d'éclairer les choix traductifs, d'une part en mettant à jour certaines conceptions et représentations du traduire conditionnant celui-ci (les imaginaires du traduire), d'autre part en explorant les 
nombreuses préconceptions, explicites ou implicites, personnelles ou générales, propres à qui traduit, et qui en influencent et modèlent le travail (les imaginaires des traducteurs et traductrices). Concept hybride, puisant tour à tour dans les sciences sociales, la psychologie ou l'esthétique, l'imaginaire constitue donc un nouvel instrument, utilisable dans toute trousse à outils traductologique, mais également une méthode éclairant d'autres perspectives épistémologiques : interculturalité, herméneutique, histoire littéraire, jusqu'à permettre d'isoler des éléments récurrents, permettant d'esquisser une potentielle cartographie des phénomènes traductifs. Il constitue également une manière de lier davantage l'approche comparatiste et l'étude des traductions.

A translation can not be read only as a clear and unidirectional exchange of signs, where one could automatically replace an other. The idea of imaginaries allows the critic to shed light on translating choices, on the one hand by studying the representations and preconceptions of the very act of translating a text (the imaginaries of translating), on the other hand by delving into every factor-be it explicit or implicit, personal or collective-that have influenced and shaped any translator's work (the imaginaries of translators). A hybrid concept which draws from social sciences, psychology or aesthetics, the imaginary becomes a new tool among the translation scholar's toolbox. Moreover, it also constitutes a method that allows to find new paths into intercultural studies, hermeneutics, literary history, so far as to allow to isolate recurring patterns of translation, which may help sketching a mapping of translation phenomenons. It is also a way to bind further the comparatist and translation studies approaches of themselves.

\section{INDEX}

Keywords : translation, translation studies, imaginary, imagination, irrational, poetics, psychology, reception studies

Mots-clés : traduction, traductologie, imaginaire, imagination, irrationnel, poétique, psychologie, réception

\section{AUTEURS}

\section{CHRISTINA BEZARI}

Université de Gand

RICCARDO RAIMONDO

Université de Zurich

THOMAS VUONG

Université Paris 13, Pléiade (EA 7338) 